\title{
Analysis of Dentoalveolar and Maxillofacial Fractures with Multidetector-Row Computed Tomography
}

\author{
Ichiro Ogura ${ }^{1, *}$, Fumi Mizuhashi ${ }^{2}$, Yoshihiro Sugawara ${ }^{3}$, Makoto Oohashi $^{4}$, Hirokazu Sekiguchi ${ }^{5}$, \\ Hisato Saegusa ${ }^{3}$
}

${ }^{1}$ Department of Oral and Maxillofacial Radiology, The Nippon Dental University School of Life Dentistry at Niigata, Niigata, Japan

${ }^{2}$ Department of Removable Prosthodontics, The Nippon Dental University School of Life Dentistry at Niigata, Niigata, Japan

${ }^{3}$ Comprehensive Dental Care, The Nippon Dental University Niigata Hospital, Niigata, Japan

${ }^{4}$ Dental Anesthesia and General Health Management, The Nippon Dental University Niigata Hospital, Niigata, Japan

${ }^{5}$ Laboratory of Dental Technology, The Nippon Dental University Niigata Hospital, Niigata, Japan

\section{Email address:}

ogura@ngt.ndu.ac.jp (I. Ogura)

${ }^{*}$ Corresponding author

\section{To cite this article:}

Ichiro Ogura, Fumi Mizuhashi, Yoshihiro Sugawara, Makoto Oohashi, Hirokazu Sekiguchi, Hisato Saegusa. Analysis of Dentoalveolar and Maxillofacial Fractures with Multidetector-Row Computed Tomography. International Journal of Medical Imaging.

Vol. 7, No. 2, 2019, pp. 40-43. doi: 10.11648/j.ijmi.20190702.12

Received: June 3, 2019; Accepted: July 4, 2019; Published: July 15, 2019

\begin{abstract}
Dentoalveolar trauma may occur after accidental falls, automotive accidents, violence, or contact sport injuries. This study aimed to investigate the prevalence of dentoalveolar and maxillofacial fractures with multidetector-row computed tomography (MDCT). Forty patients with dentoalveolar and maxillofacial fractures underwent MDCT. Mandibular fractures were classified into four types: median, paramedian, angle and condylar types. Midface fractures were classified into four types: Le Fort I-III and zygomatic maxillary complex types. Statistical analysis of the relationship between prevalence of dentoalveolar fractures and maxillofacial fracture locations was performed using Chi-square test with Fisher's exact test. A p-value less than 0.05 was considered statistically significant. The prevalence of dentoalveolar fractures was $15.0 \%$ (6/40 cases) of all patients with maxillofacial fractures. The mean age of patients with and without dentoalveolar fractures in maxillofacial fractures were 39.8 years and 54.7 years, respectively. Regarding cause of injury, the prevalence of maxillofacial fractures with dentoalveolar fractures were $18.8 \%$ of accidental falls and $0 \%$ of contact sport injuries and automotive accidents. There was no significant relationship between prevalence of dentoalveolar and maxillofacial fractures. The results suggest that the prevalence of dentoalveolar and maxillofacial fractures are related to the age and cause of injury.
\end{abstract}

Keywords: Dentoalveolar Fractures, Mandibular Fractures, Midfacial Fractures, Computed Tomography

\section{Introduction}

Dentoalveolar trauma may occur after accidental falls, automotive accidents, violence, contact sport injuries or accidents associated with the use of wheeled devices such as bicycles, skateboards, scooters or roller skates, both in children and adults [1-2]. Trauma may results in tooth fracture and/or alveolar process injury. When the dental fracture happens in the crown, it is clinically diagnosed and the periapical radiograph is used to evaluate its extension and proximity to the pulp [3]. However, in cases of root fracture, periapical radiograph or cone-beam computed tomography
(CBCT) images may be used to confirm the fracture and observe the tooth and adjacent alveolar bone.

Radiographic evaluation is an indispensable tool for diagnosing fractures and traumatic injuries to the maxillofacial complex [4]. Multidetector-row computed tomography (MDCT) offers superior soft tissue characterization and is useful for diagnosis of odontogenic and nonodontogenic cysts and tumors, fibro-osseous lesions, inflammatory, malignancy, metastatic lesions, developmental abnormalities, and maxillofacial trauma [5]. Fracture morphology of maxillofacial trauma is often complex, so the clinicians should be familiar with the imaging findings [6]. In 
recent years, MDCT with multiplanar reformation (MPR) and three-dimensional (3D) images has become a standard part of the assessment of maxillofacial injury because of the exquisite sensitivity of this imaging technique for fracture [7-14]. However, to the best of our knowledge, analysis of dentoalveolar and maxillofacial fractures with MDCT have not been reported in the literature. The aim of this study was to investigate the prevalence of dentoalveolar and maxillofacial fractures with MDCT.

\section{Materials and Methods}

This prospective study was approved by the ethics committee of our institution. After obtaining written informed consent, 40 patients (28 male, 12 female; age 15-91 years, mean age 52.5 years) with dentoalveolar and maxillofacial fractures who underwent MDCT at our university hospital from October 2016 to May 2019.

MDCT imaging was performed with a 16-MDCT scanner (Aquilion TSX-101A; Canon Medical Systems, Otawara, Japan) using the maxillofacial protocol at our hospital: tube voltage, $120 \mathrm{kV}$; tube current, $150 \mathrm{mAs}$; field of view, $240 \times 240 \mathrm{~mm}$; and rotation time, $0.50 \mathrm{~s}$. The protocol consisted of axial acquisition $(0.50 \mathrm{~mm})$ with axial, coronal, and sagittal MPR and 3D images.

The MDCT images were independently evaluated by two oral and maxillofacial radiologists and any discrepancies were resolved by consensus. Mandibular fractures were classified into four types: median, paramedian, angle and condylar types [7]. Midface fractures were classified into four types: Le Fort I-III and zygomatic maxillary complex types [12].

Age was compared between with and without dentoalveolar fractures using the Mann-Whitney U test. Statistical analysis of the relationship between prevalence of dentoalveolar fractures and gender, cause of injury and fracture locations with MDCT was performed using Chi-square test with Fisher's exact test. These analyses were performed with the statistical package SPSS version 26.0 (IBM Japan, Tokyo, Japan). A p-value less than 0.05 was considered statistically significant.

\section{Results}

Table 1 shows patient distribution according to location of maxillofacial fractures with/without dentoalveolar fractures. The prevalence of dentoalveolar fractures was 15.0\% (6/40 cases) of all patients with maxillofacial fractures (Figure 1 and Figure 2). Table 2 shows relationship between dentoalveolar and maxillofacial fractures. The mean age of patients with and without dentoalveolar fractures in maxillofacial fractures were 39.8 years and 54.7 years, respectively. Regarding cause of injury, the prevalence of maxillofacial fractures with dentoalveolar fractures were $18.8 \%$ of accidental falls and $0 \%$ of contact sport injuries and automotive accidents. There was no significant relationship between prevalence of dentoalveolar and maxillofacial fractures.

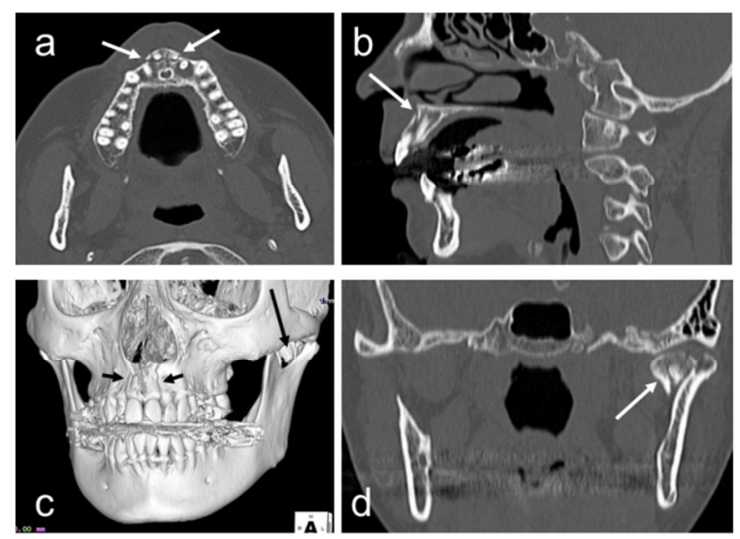

Figure 1. Dentoalveolar and mandibular condylar fractures in a 54-year-old female. (a) Axial CT image shows dentoalveolar fractures (arrows). (b) Sagittal CT image shows dentoalveolar fractures (arrow). (c) 3D-CT image shows dentoalveolar fractures (short arrows) and mandibular condylar fractures (long arrow). (d) Coronal CT image shows mandibular condylar fractures (arrow)
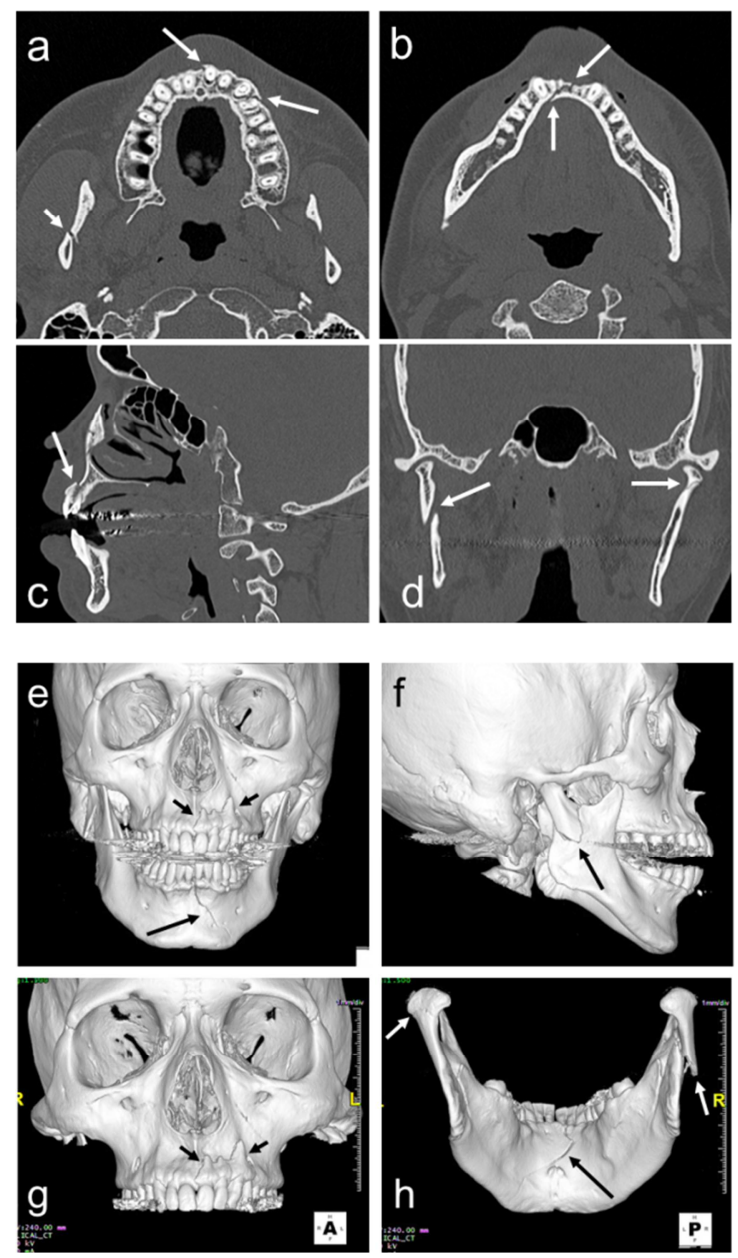

Figure 2. Dentoalveolar and mandibular median and bi-condylar fractures in a 43-year-old male. (a) Axial CT image shows dentoalveolar fractures (long arrows) and mandibular condylar fractures (short arrow). (b) Axial CT image shows mandibular median fractures (arrows). (c) Sagittal CT image shows dentoalveolar fractures (arrow). (d) Coronal CT image shows mandibular bi-condylar fractures (arrows). (e) 3D-CT image shows dentoalveolar fractures (short arrows) and mandibular median fractures (long arrow). (f) $3 D$-CT image shows mandibular condylar fractures (arrow). (g) 3D-CT image shows dentoalveolar fractures (arrows). (h) 3D-CT image shows mandibular median (black arrow) and bi-condylar (white arrows) fractures. 
Table 1. Patient distribution according to location of maxillofacial fractures with/without dentoalveolar fractures.

\begin{tabular}{|c|c|c|c|}
\hline \multirow{3}{*}{ Location of maxillofacial fractures with MDCT } & \multicolumn{3}{|c|}{ Patients with/without dentoalveolar fractures } \\
\hline & With & Without & Total \\
\hline & $(n=6,15.0 \%)$ & $(n=34,85.0 \%)$ & $(n=40,100 \%)$ \\
\hline Mandible & & & $33(82.5 \%)$ \\
\hline Condylar & 2 & 13 & 15 \\
\hline Condylar and median & 1 & 5 & 6 \\
\hline Condylar and paramedian & 0 & 2 & 2 \\
\hline Median and angle & 0 & 5 & 5 \\
\hline Median and paramedian & 1 & 0 & 1 \\
\hline Paramedian and angle & 0 & 1 & 1 \\
\hline Median & 0 & 1 & 1 \\
\hline Paramedian & 0 & 1 & 1 \\
\hline Angle & 1 & 0 & 1 \\
\hline Midface & & & $7(17.5 \%)$ \\
\hline Zygomatic maxillary complex & 1 & 2 & 3 \\
\hline Le Fort I & 0 & 3 & 3 \\
\hline Le Fort II & 0 & 1 & 1 \\
\hline
\end{tabular}

MDCT: multidetector-row computed tomography

Table 2. Relationship between dentoalveolar and maxillofacial fractures.

\begin{tabular}{|c|c|c|c|c|}
\hline & \multicolumn{4}{|c|}{ Patients with/without dentoalveolar fractures } \\
\hline & With & Without & Total & p-value \\
\hline Number of patients & $6(15.0 \%)$ & $34(85.0 \%)$ & 40 & \\
\hline Age (years) & & & & 0.109 \\
\hline Mean \pm SD & $39.8 \pm 15.9$ & $54.7 \pm 23.5$ & $52.5 \pm 23.0$ & \\
\hline Range & $17-60$ & $15-91$ & $15-91$ & \\
\hline Gender & & & & 1.000 \\
\hline Male & $4(14.3 \%)$ & $24(85.7 \%)$ & 28 & \\
\hline Cause of injury & & & & 0.318 \\
\hline Accidental falls & $6(18.8 \%)$ & $26(81.2 \%)$ & 32 & \\
\hline Contact sport injuries & $0(0 \%)$ & $5(100 \%)$ & 5 & \\
\hline Automotive accidents & $0(0 \%)$ & $3(100 \%)$ & 3 & \\
\hline Fracture location with MDCT & & & & \\
\hline Condylar & $3(13.0 \%)$ & $20(87.0 \%)$ & 23 & 1.000 \\
\hline Median & $2(15.4 \%)$ & $11(84.6 \%)$ & 13 & 1.000 \\
\hline Paramedian & $1(20.0 \%)$ & $4(80.0 \%)$ & 5 & 1.000 \\
\hline
\end{tabular}

SD: standard deviation; MDCT: multidetector-row computed tomography

\section{Discussion}

Kobayashi-Velasco et al [1] demonstrated that the protocol for the diagnosis of root and alveolar fractures was voxel 0.20 $\mathrm{mm}, 400$ frames and radiation exposure $5.6 \mathrm{mGy}$ in CBCT. Furthermore, Kobayashi-Velasco et al [3] showed that periapical radiograph showed poorer results than CBCT for the diagnosis of alveolar fractures. However, in recent years, MDCT with MPR and 3D images has become a standard part of the assessment of maxillofacial injury because of the exquisite sensitivity of this imaging technique for fracture. Therefore, we analyzed dentoalveolar and maxillofacial fractures with MDCT.

In fracture of the mandible or maxilla, the fracture involves the base of the mandible or maxilla and the alveolar process [4]. Gupta et al [2] showed that different types of tooth injuries associated with facial bone fracture found more in females and maxillary teeth. In this study, the mean age of patients with and without dentoalveolar fractures in maxillofacial fractures were 39.8 years and 54.7 years, respectively. We consider that the patients with dentoalveolar fractures found more in younger.

Regarding patient distribution according to location of maxillofacial fractures with/without dentoalveolar fractures, the prevalence of dentoalveolar fractures was $15.0 \%(6 / 40$ cases) of all patients with maxillofacial fractures in this study. Furthermore, we showed no relationship between the prevalence of dentoalveolar and maxillofacial fractures. We consider that the prevalence of dentoalveolar fractures are not related to the maxillofacial fracture locations.

A limitation of this study was the small number of dentoalveolar and maxillofacial fractures. Additionally, logistic regression regarding the comparison between patients with and without dentoalveolar fractures was not used to determine the independent predictors of maxillofacial fractures in this group of patients. 


\section{Conclusions}

This study investigated the prevalence of dentoalveolar and maxillofacial fractures with MDCT. The prevalence of dentoalveolar fractures was $15.0 \%$ of all patients with maxillofacial fractures. The mean age of patients with and without dentoalveolar fractures in maxillofacial fractures were 39.8 years and 54.7 years, respectively. Regarding cause of injury, the prevalence of maxillofacial fractures with dentoalveolar fractures were $18.8 \%$ of accidental falls and $0 \%$ of contact sport injuries and automotive accidents. The results suggest that the prevalence of dentoalveolar and maxillofacial fractures are related to the age and cause of injury.

\section{Acknowledgements}

This work was supported by NDU Grants N-19005.

\section{References}

[1] Kobayashi-Velasco S, Salineiro FCS, Gialain IO, Cavalcanti MGP. Diagnosis of alveolar and root fractures in macerated canine maxillae: a comparison between two different CBCT protocols. Dentomaxillofac Radiol. 2017; 46: 20170037.

[2] Gupta M, Das D, Soodan K, Singh C. Dental injuries in patients associated with fracture of facial bones. Oral Maxillofac Surg. 2019; 23: 63-9.

[3] Kobayashi-Velasco S, Salineiro FC, Gialain IO, Cavalcanti MG. Diagnosis of alveolar and root fractures: an in vitro study comparing CBCT imaging with periapical radiographs. J Appl Oral Sci. 2017; 25: 227-33.

[4] Alimohammadi R. Imaging of dentoalveolar and jaw trauma. Radiol Clin North Am. 2018; 56: 105-24.
[5] Gohel A, Oda M, Katkar AS, Sakai O. Multidetector row computed tomography in maxillofacial imaging. Dent Clin North Am. 2018; 62: 453-65.

[6] Ogura I, Sasaki Y, Kaneda T. Multidetector computed tomography of maxillofacial fractures. Jpn Dent Sci Rev. 2014; 50: $86-90$

[7] Ogura I, Kaneda T, Mori S, Sekiya K, Ogawa H, Tsukioka T. Characterization of mandibular fractures using 64-slice multidetector CT. Dentomaxillofac Radiol. 2012; 41: 392-5.

[8] Ogura I, Sasaki Y, Kaneda T. Analysis of mandibular condylar and glenoid fossa fractures with computed tomography. Eur Radiol. 2014; 24: 902-6.

[9] Ohki T, Ogura I. Characteristic multidetector computed tomography findings of maxillofacial fractures resulting from falls in the elderly. Int J Oral-Med Sci. 2014; 13: 1-5.

[10] Honda I, Ogura I. Characteristic multidetector CT findings of mandibular fractures: comparison with falls and violence. Int J Oral-Med Sci. 2014; 13: 53-8.

[11] Ogura I, Kaneda T, Sasaki Y, Buch K, Sakai O. Prevalence of temporal bone fractures in patients with mandibular fractures using multidetector-row CT. Clin Neuroradiol. 2015; 25: $137-41$.

[12] Ogura I, Kawashima Y, Muramatsu T, Ito K, Kaneda T. Characteristic computed tomographic findings of midface fractures relative to the cause of injury: a fall or violence. Oral Radiol. 2015; 31: 149-54.

[13] Ogura I, Hara Y, Tokunaga S, Kaneda T. Mandibular coronoid process fractures: prevalence and characteristic multidetector CT findings. Oral Sci Int. 2016; 13: 33-6.

[14] Ogura I, Hirahara N, Muraoka H, Fukuda T, Hara Y, Tokunaga S. Characteristics of maxillofacial fractures in elderly patients compared with young patients. Int J Oral-Med Sci. 2016; 15: $10-6$. 